\title{
Central limit theorem for linear processes generated by IID random variables under the sub-linear expectation
}

\author{
LIU Wei ZHANG Yong*
}

\begin{abstract}
In this paper, we investigate the central limit theorem and the invariance principle for linear processes generated by a new notion of independently and identically distributed (IID) random variables for sub-linear expectations initiated by Peng [19]. It turns out that these theorems are natural and fairly neat extensions of the classical Kolmogorov's central limit theorem and invariance principle to the case where probability measures are no longer additive.
\end{abstract}

\section{$\S 1 \quad$ Introduction}

Based on the framework of classical probability theory established by Kolmogorov, probability and expectation are both linear. Classical limit theorems only hold in the case of model certainty. Motivated by modeling uncertainty in practice, Peng ( [16]- [21]) introduced a new notion of sub-linear expectation. As an alternative to the traditional probability/expectation, capacity/sub-linear expectation has been studied in many fields such as statistics, finance, economics, and measures of risk (see Denis and Martini [9], Gilboa [7], Marinacci [14], Peng [16] etc.). Peng ( [16]- [21]) introduced the reasonable framework of the sub-linear expectation of random variables in a general function space by relaxing the linear property of the classical linear expectation to the sub-additivity and positive homogeneity. In addition, the notion of independent identically distributed (IID for short) random variable under sub-linear expectation is introduced. He also introduced the notion of G-normal distribution and G-Brownian motion as the counterpart of normal distribution and Brownian motion in linear case, respectively. Gexpectation space is the most important sub-linear expectation space introduced by Peng [18], which takes the role of Wiener space in classical.

\footnotetext{
Received: 2019-08-16. Revised: 2019-12-19.

MR Subject Classification: 60F05, 60F 17.

Keywords: central limit theorem, invariance principle, IID random variables, sub-linear expectation, linear process.

Digital Object Identifier(DOI): https://doi.org/10.1007/s11766-021-3882-7.

Supported by the National Natural Science Foundation of China (11771178), the Science and Technology Development Program of Jilin Province (20170101152JC), the Science and Technology Program of Jilin Educational Department during the "13th Five-Year" Plan Period (JJKH20200951KJ) and Fundamental Research Funds for the Central Universities.

* Corresponding author.

(C)The Author(s) 2021.
} 
The classical central limit theorem (CLT for short) is a fundamental result in probability theory. In recent years, the limit theorems of the sub-linear expectation have received more and more attention and extensive research. Peng [19] initiated the CLT for sub-linear expectation for a sequence of independent identical distribution random variables with finite $(2+\alpha)$-moments for some $\alpha>0$. The CLT for sub-linear expectation has gotten considerable development. Hu and Zhang [5] obtained a CLT for capacity. Hu [6] extended Pengs CLT by weakening the assumptions of test functions. Hu and Zhou [24] presented some multi-dimensional CLTs without assumption of identical distribution. Li [23] proved a CLT for sub-linear expectation for a sequence of $\mathrm{m}$-dependent random variables. Rokhlin [3] gave a CLT under the Lindeberg condition under classical probability with variance uncertainty. Zhang [10] gained a CLT for sub-linear expectation under a moment condition weaker than $(2+\alpha)$-moments. Zhang [11] established a martingale CLT and functional CLT for sub-linear expectation under the Lindeberg condition. $\mathrm{Wu}$ and Chen [15] obtained a general invariance principle of G-Brownian motion for the law of the iterated logarithm for continuous bounded independent and identically distributed random variables in G-expectation space. Zhang [12] proved a new Donsker's invariance principle for independent and identically distributed random variables under the sub-linear expectation.

As we all know, the linear processes are specially important in time series analysis and they arise from a wide variety of contexts (see, e.g., Hannan [4]). Applications to economics, engineering and physical sciences are extremely broad and a vast amount of literature is devoted to the study on linear processes under a variety of circumstances.

A natural question is: Can the classical CLT and invariance principle for linear processes be generated by IID random variables under Peng's framework? The main purpose of this paper is to establish a central limit theorem and an invariance principle for linear processes generated by IID random variables under the sub-linear expectation. The discovered phenomenon plays the same important role in the theory of sub-linear expectation as that of the CLT and invariance principle in classic probability theory. In the classical case, the CLT of partial sum is established by decomposing the linear process. We will find that this way is also valid for proving CLT for linear process in the sub-linear expectation space, though there are some differences. The main difference is that the probability space $(\Omega, \mathcal{F}, P)$ of Kolmogorov probability axiom system is replaced by the sub-linear expectation space $(\Omega, \mathcal{H}, \widehat{\mathbb{E}})$. Intuitively, it is sub-linear expectation that plays a decisive role in our proof.

Our paper is organized as follows: we introduce some basic settings and notations in Section 2. In Section 3, based on the framework introduced by Peng ( [16]- [21]), the main results and proofs are given. C denotes a positive constant, which may take different values whenever it appears in different expressions.

\section{$\S 2 \quad$ Basic settings and lemmas}

We use the framework and notation of Peng ( [16]- [21]). Let $(\Omega, \mathcal{F})$ be a given measurable space. Let $\mathcal{H}$ be a linear space of real functions defined on $\Omega$ such that if $X_{1}, X_{2}, \ldots, X_{n} \in \mathcal{H}$ 
then $\varphi\left(X_{1}, X_{2}, \ldots, X_{n}\right) \in \mathcal{H}$ for each $\varphi \in C_{l, L i p}\left(\mathbb{R}^{n}\right)$ where $\varphi \in C_{l, \text { Lip }}\left(\mathbb{R}^{n}\right)$ denotes the linear space of local Lipschitz continuous functions $\varphi$ satisfying

$$
|\varphi(x)-\varphi(y)| \leq C\left(1+|x|^{m}+|y|^{m}\right)|x-y|, \quad \forall x, y \in \mathbb{R}^{n},
$$

for some $C>0, m \in \mathbb{N}$ depending on $\varphi$. $\mathcal{H}$ contains all $I_{A}$ where $A \in \mathcal{F}$. We also denote $\varphi \in C_{b, L i p}\left(\mathbb{R}^{n}\right)$ as the linear space of bounded Lipschitz continuous functions $\varphi$ satisfying

$$
|\varphi(x)-\varphi(y)| \leq C|x-y|, \quad \forall x, y \in \mathbb{R}^{n},
$$

for some $C>0$.

Definition 2.1 A function $\widehat{\mathbb{E}}: \mathcal{H} \rightarrow \overline{\mathbb{R}}$ is said to be a sub-linear expectation if it satisfies for $\forall X, Y \in \mathcal{H}$,

(a) Monotonicity: $X \geq Y$ implies $\widehat{\mathbb{E}}[X] \geq \widehat{\mathbb{E}}[Y]$.

(b) Constant preserving: $\widehat{\mathbb{E}}[c]=c, \forall c \in \mathbb{R}$.

(c) Sub-additivity: $\widehat{\mathbb{E}}[X+Y] \leq \widehat{\mathbb{E}}[X]+\widehat{\mathbb{E}}[Y]$ whenever $\widehat{\mathbb{E}}[X]+\widehat{\mathbb{E}}[Y]$ is not of the from $+\infty-\infty$ or $-\infty+\infty$.

(d) Positive homogeneity: $\widehat{\mathbb{E}} \lambda X]=\lambda \widehat{\mathbb{E}}[X], \forall \lambda>0$.

Here $\overline{\mathbb{R}}=[-\infty,+\infty]$. The triple $(\Omega, \mathcal{H}, \widehat{\mathbb{E}})$ is called a sub-linear expectation space. Give a sublinear expectation $\widehat{\mathbb{E}}$, let us denote the conjugate expectation $\widehat{\mathcal{E}}$ of $\widehat{\mathbb{E}}$ by $\widehat{\mathcal{E}}[X]:=-\widehat{\mathbb{E}}[-X], \forall X \in$ $\mathcal{H}$.

Remark 2.1. (i) The sub-linear expectation $\widehat{\mathbb{E}}[\cdot]$ satisfies translation invariance: $\widehat{\mathbb{E}}[X+c]=$ $\widehat{\mathbb{E}}[X]+c, \forall c \in \mathbb{R}$.

(ii) From the definition, it is easily shown that $\widehat{\mathcal{E}}[X] \leq \widehat{\mathbb{E}}[X]$ and $\widehat{\mathbb{E}}[X-Y] \geq \widehat{\mathbb{E}}[X]-\widehat{\mathbb{E}}[Y], \forall X, Y \in$ $\mathcal{H}$ with $\widehat{\mathbb{E}}[Y]$ being finite.

(iii) Furthermore, if $\widehat{\mathbb{E}}[|X|]$ is finite, then $\widehat{\mathbb{E}}[X]$ and $\widehat{\mathcal{E}}[X]$ are both finite. We also call $\widehat{\mathbb{E}}[X]$ and $\widehat{\mathcal{E}}[X]$ the upper expectation and lower expectation of $X$, respectively.

Definition 2.2 (Independence) In a sub-linear expectation space $(\Omega, \mathcal{H}, \widehat{\mathbb{E}})$, a random vector $Y=\left(Y_{1}, \ldots, Y_{n}\right)\left(Y_{i} \in \mathcal{H}\right)$ is said to be independent to another random vector $X=$ $\left(X_{1}, \ldots, X_{m}\right)\left(X_{i} \in \mathcal{H}\right)$ under $\widehat{\mathbb{E}}$ if for each test function $\varphi \in C_{l, L i p}\left(\mathbb{R}^{m} \times \mathbb{R}^{n}\right)$ we have

$$
\widehat{\mathbb{E}}[\varphi(X, Y)]=\widehat{\mathbb{E}}\left[\left.\widehat{\mathbb{E}}[\varphi(x, Y)]\right|_{x=X}\right],
$$

whenever $\bar{\varphi}(x):=\widehat{\mathbb{E}}[|\varphi(x, Y)|]<\infty$ for all $x$ and $\widehat{\mathbb{E}}[|\bar{\varphi}(x)|]<\infty$.

$\left\{X_{n}, n \geq 1\right\}$ is said to be a sequence of independent random variables if $X_{n+1}$ is independent of $\left(X_{1}, \ldots, X_{n}\right)$ for each $n \geq 1$.

As shown by Peng [19], it is important to note that under sub-linear expectations the condition that $Y$ is independent to $X$ does not imply automatically that $X$ is independent to $Y$.

Remark 2.2. (Zhang [13]) From the definition of independence, it is easily seen that, if $Y$ is independent to $X$, and $X, Y \in \mathcal{H}, X \geq 0, \widehat{\mathbb{E}}[Y] \geq 0$, then

$$
\mathbb{E}[X Y]=\widehat{\mathbb{E}}[X] \widehat{\mathbb{E}}[Y],
$$


Furthermore, if $Y$ is independent to $X$ and $0 \leq X, Y \in \mathcal{H}$, then

$$
\widehat{\mathcal{E}}[X Y]=\widehat{\mathcal{E}}[X] \widehat{\mathcal{E}}[Y] .
$$

Let $\mathbf{X}$ be an $n$-dimensional random variable on a sub-linear expectation space $(\Omega, \mathcal{H}, \widehat{\mathbb{E}})$. We define a functional on $C_{l, L i p}\left(\mathbb{R}^{n}\right)$ such that

$$
\mathbb{F}_{X}[\varphi]:=\widehat{\mathbb{E}}[\varphi(X)], \varphi \in C_{l, L i p}\left(\mathbb{R}^{n}\right) \rightarrow \mathbb{R},
$$

Then $\mathbb{F}_{X}[\cdot]$ can be regard as the distribution of $\mathbf{X}$ under $\widehat{\mathbb{E}}$ and it characterizes the uncertainty of the distribution of $\mathbf{X}$.

Definition 2.3 (Identical distribution) Let $X_{1}$ and $X_{2}$ be two n-dimensional random vectors defined respectively in sub-linear expectation spaces $\left(\Omega_{1}, \mathcal{H}_{1}, \widehat{\mathbb{E}}_{1}\right)$ and $\left(\Omega_{2}, \mathcal{H}_{2}, \widehat{\mathbb{E}}_{2}\right)$. They are called identically distributed, denoted by $X_{1} \stackrel{d}{=} X_{2}$, if

$$
\widehat{\mathbb{E}}_{1}\left[\varphi\left(X_{1}\right)\right]=\widehat{\mathbb{E}}_{2}\left[\varphi\left(X_{2}\right)\right], \forall \varphi \in C_{l, L i p}\left(\mathbb{R}^{n}\right),
$$

whenever the sub-expectations are finite. A sequence of random variables $\left\{X_{n}, n \geq 1\right\}$ is said to be identically distributed if $X_{i} \stackrel{d}{=} X_{1}$ for each $i \geq 1$.

Definition 2.4 (IID random variables) A sequence of random variables $\left\{X_{n}, n \geq 1\right\}$ is said to be independent and identically distributed (IID), if $X_{i} \stackrel{d}{=} X_{1}$ and $X_{i+1}$ is independent to $\left(X_{1}, \ldots, X_{i}\right)$ for each $i \geq 1$.

Definition 2.5 (G-normal distribution) A random variable $\xi \in \mathcal{H}$ under sub-linear expectation $\widehat{\mathbb{E}}$ with $\bar{\sigma}^{2}=\widehat{\mathbb{E}}\left[\xi^{2}\right], \underline{\sigma}^{2}=-\widehat{\mathbb{E}}\left[-\xi^{2}\right]$ is called G-normal distribution, denoted by $\mathcal{N}\left(0 ;\left[\underline{\sigma}^{2}, \bar{\sigma}^{2}\right]\right)$, if for any function $\varphi \in C_{l, L i p}(\mathbb{R}), u(t . x):=\widehat{\mathbb{E}}[\varphi(x+\sqrt{t} \xi)],(t, x) \in[0, \infty) \times \mathbb{R}$, then $u$ is the unique viscosity solution of PDE:

$$
\left\{\begin{array}{l}
\partial_{t}-G\left(\partial_{x x} u\right)=0, \\
\left.u\right|_{t=0}=\varphi
\end{array}\right.
$$

where $G(\alpha)=\frac{1}{2}\left(\bar{\sigma}^{2} \alpha^{+}-\underline{\sigma}^{2} \alpha^{-}\right)$and $\alpha^{+}:=\max (\alpha, 0), \alpha^{-}:=(-\alpha)^{+}$.

Remark 2.3. The G-normal distributed random variables $\xi$ satisfies $a \xi+b \bar{\xi} \stackrel{d}{=} \sqrt{a^{2}+b^{2}} \xi, \forall a, b \geq$ 0 , where $\bar{\xi} \stackrel{d}{=} \xi$ and $\bar{\xi}$ is independent of $\xi$. This implies $\widehat{\mathbb{E}}[\xi]=\widehat{\mathbb{E}}[-\xi]=0$.

Let $C[0,1]$ be a function space of continuous functions on $[0,1]$ equipped with the supernorm $\|x\|=\sup _{0 \leq t \leq 1}|x(t)|$ and $C_{b}\{C[0,1]\}$ is the set of bounded continuous functions $h(x)$ : $C[0,1] \rightarrow \mathbb{R}$. The modulus of the continuity of an element $x \in C[0,1]$ is defined by

$$
\omega_{\delta}(x)=\sup _{|t-s|<\delta}|x(t)-x(s)| .
$$

Denis et al. [2] showed that there is a sub-linear expectation space $(\widetilde{\Omega}, \widetilde{\mathcal{H}}, \widetilde{\mathbb{E}})$ with $\widetilde{\Omega}=$ $C[0,1]$ and $C_{b}\{C[0,1]\} \subset \widetilde{\mathcal{H}}$ such that $\widetilde{\mathbb{E}}$ is countably sub-additive, and the canonical process $W(t)(\omega)=\omega_{t}(\omega \in \widetilde{\Omega})$ is a G-Brownian motion with $W(1) \sim N\left(0 ;\left[\underline{\sigma}^{2}, \bar{\sigma}^{2}\right]\right)$ under $\widetilde{\mathbb{E}}$, i.e., for all $0 \leq t_{1}<\ldots<t_{n} \leq 1, \varphi \in C_{b, l i p}\left(\mathbb{R}^{n}\right)$,

$$
\widetilde{\mathbb{E}}\left[\varphi\left(W\left(t_{1}\right), \ldots, W\left(t_{n-1}\right), W\left(t_{1}\right)-W\left(t_{n-1}\right)\right)\right]=\widetilde{\mathbb{E}}\left[\psi\left(W\left(t_{1}\right), \ldots, W\left(t_{n-1}\right)\right)\right],
$$


where $\psi\left(x_{1}, \ldots, x_{n-1}\right)=\widetilde{\mathbb{E}}\left[\varphi\left(x_{1}, \ldots, x_{n-1}, \sqrt{t_{n}-t_{n-1}} W(1)\right)\right]$.

Denis et al. [2] also showed the following representation of the G-Brownian motion (c.f. [2, Proposition 49]).

Lemma 2.1. Let $(\Omega, \mathcal{F}, P)$ be a probability measure space and $[B(t)]_{t \geq 0}$ is a P-Brownian motion. Then for all $\varphi \in C_{b}(\widetilde{\Omega})$,

where

$$
\widetilde{\mathbb{E}}[\varphi(W)]=\sup _{\theta \in \Theta} E_{p}\left[\varphi\left(W_{\theta}\right)\right], \quad W_{\theta}(t)=\int_{0}^{1} \theta(s) d B(s),
$$

$$
\begin{aligned}
& \Theta=\left\{\theta: \theta(t) \text { is } \mathcal{F}_{t}-\text { adapted process such that } \underline{\sigma} \leq \theta(s) \leq \bar{\sigma}\right\} \\
& \mathcal{F}_{t}=\sigma\{B(s): 0 \leq s \leq t\} \vee \mathcal{N}, \mathcal{N} \text { is the collection of P-null subsets. }
\end{aligned}
$$

The following two lemmas are useful for the proof of Lemma 3.1.

Lemma 2.2. (Linero and Rosalsky [1]) (Kronecker Lemma). Let $\left\{x_{n}, n \geq 1\right\}$ and $\left\{b_{n}, n \geq 1\right\}$ be sequences of real numbers with $0<b_{n} \uparrow \infty$. If the series $\sum_{k=1}^{\infty} \frac{x_{k}}{b_{k}}$ converges, then

$$
\lim _{n \rightarrow \infty} \sum_{k=1}^{n} \frac{x_{k}}{b_{n}}=0
$$

Lemma 2.3. (Xu and Zhang [8])(Rosnethal's inequality). Let $\left\{X_{n}, n \geq 1\right\}$ be a sequence of independent random variables on the sub-linear expectation space $(\Omega, \mathcal{H}, \widehat{\mathbb{E}})$, and denote $S_{k}=$ $X_{1}+X_{2}+\ldots+X_{k}, S_{0}=0$. If both the upper expectation $\widehat{\mathbb{E}}\left[X_{k}\right]$ and the lower expectation $\widehat{\mathcal{E}}\left[X_{k}\right]$ are zeros, $k=1,2, \ldots$, then

$$
\widehat{\mathbb{E}}\left[\max _{k \leq n}\left|S_{k}\right|^{p}\right] \leq 2 \sum_{k=1}^{n} \widehat{\mathbb{E}}\left[\left|X_{k}\right|^{p}\right], \quad \text { for } 1 \leq p \leq 2 .
$$

Zhang [10] obtained the following central limit theorem for independent and identically distributed random variables with only finite variances, which improves the central limit theorem of Peng [19] and is of independent interest. Let $\left\{X_{n}, n \geq 1\right\}$ be a sequence of random variables in $(\Omega, \mathcal{H}, \widehat{\mathbb{E}})$, set $S_{n}=0, S_{n}=\sum_{k=1}^{n} X_{k}$.

Theorem 2.1. Suppose that $\left\{X_{n}, n \geq 1\right\}$ is a sequence of independent and identically distributed random variables with $\widehat{\mathbb{E}}\left[X_{1}\right]=\widehat{\mathbb{E}}\left[-X_{1}\right]=0$ and $\lim _{c \rightarrow \infty} \widehat{\mathbb{E}}\left[\left(X_{1}^{2}-c\right)^{+}\right]=0$. Write $\bar{\sigma}^{2}=\widehat{\mathbb{E}}\left[X_{1}^{2}\right]$ and $\underline{\sigma}^{2}=\widehat{\mathcal{E}}\left[X_{1}^{2}\right]$. Then for any continuous function $\varphi$ satisfying $|\varphi(x)| \leq C\left(1+x^{2}\right)$,

$$
\lim _{n \rightarrow \infty} \widehat{\mathbb{E}}\left[\varphi\left(\frac{S_{n}}{\sqrt{n}}\right)\right]=\widetilde{\mathbb{E}}[\varphi(\xi)],
$$

where $\xi \sim \mathcal{N}\left(0 ;\left[\underline{\sigma}^{2}, \bar{\sigma}^{2}\right]\right)$ under $\widetilde{\mathbb{E}}$. Furthermore, if $p>2$ and $\widehat{\mathbb{E}}\left[\left|X_{1}\right|^{p}\right]<\infty$, then (1) holds for any continuous function $\varphi$ satisfying $|\varphi(x)| \leq C\left(1+|x|^{p}\right)$.

Zhang [12] obtained a new Donsker's invariance principle for independent and identically distributed random variables under the sub-linear expectation. The sequence $\left\{X_{n}, n \geq 1\right\}$ of the random variables is considered in $(\Omega, \mathcal{H}, \widehat{\mathbb{E}})$ and Brownian motions are considered in 
$(\widetilde{\Omega}, \widetilde{\mathcal{H}}, \widetilde{\mathbb{E}})$. And suppose $\left\{X_{n}, n \geq 1\right\}$ is a sequence of independent and identically distributed random variables in $(\Omega, \mathcal{H}, \widehat{\mathbb{E}})$ with $\widehat{\mathbb{E}}\left[X_{1}\right]=\widehat{\mathbb{E}}\left[-X_{1}\right]=0, \bar{\sigma}^{2}=\widehat{\mathbb{E}}\left[X_{1}^{2}\right]$ and $\underline{\sigma}^{2}=\widehat{\mathcal{E}}\left[X_{1}^{2}\right]$, and suppose $W(t)$ is a G-Brownian motion on $(\widetilde{\Omega}, \widetilde{\mathcal{H}}, \widetilde{\mathbb{E}})$ with $W(1) \sim \mathcal{N}\left(0 ;\left[\underline{\sigma}^{2}, \bar{\sigma}^{2}\right]\right)$. Denote $S_{0}=0$, $S_{n}=\sum_{k=1}^{n} X_{k}$.

Define the $C[0,1]$-valued random variable $W_{n}$ by setting

$$
W_{n}(t)=\left\{\begin{array}{l}
S_{k} / \sqrt{n}, \text { if } t=k / n(k=0,1, \ldots, n) ; \\
\text { extended by linear interpolation in each interval } \\
{[[k-1] / n, k / n] .}
\end{array}\right.
$$

Theorem 2.2. Suppose $\widehat{\mathbb{E}}\left[\left(\varepsilon_{1}^{2}-b\right)^{+}\right]=0$ as $b \rightarrow \infty$. Then for all bounded continuous function $\varphi: C[0,1] \rightarrow \mathbb{R}$

$$
\widehat{\mathbb{E}}\left[\varphi\left(W_{n}\right)\right] \rightarrow \widetilde{\mathbb{E}}[\varphi(W)] .
$$

\section{$\S 3$ Main results and proofs}

In the sequel of this paper, consider a linear process of the form

$$
X_{t}=\sum_{j=-\infty}^{\infty} \alpha_{j} \varepsilon_{t-j}, \quad t \geq 1
$$

defined on a sub-linear expectation space $(\Omega, \mathcal{H}, \widehat{\mathbb{E}})$, where $\left\{\varepsilon_{j}, j \in \mathbb{Z}\right\}$ is a sequence of IID random variables with $\widehat{\mathbb{E}}\left[\varepsilon_{1}\right]=\widehat{\mathbb{E}}\left[-\varepsilon_{1}\right]=0, \bar{\sigma}^{2}=\widehat{\mathbb{E}}\left[\varepsilon_{1}^{2}\right]$ and $\underline{\sigma}^{2}=\widehat{\mathcal{E}}\left[\varepsilon_{1}^{2}\right],\left\{\alpha_{j}, j \in \mathbb{Z}\right\}$ is a sequence of real numbers with

$$
\sum_{j=-\infty}^{\infty}\left|\alpha_{j}\right|<\infty
$$

Define $A=\sum_{j=-\infty}^{\infty} \alpha_{j} \neq 0$,

$$
\begin{gathered}
T_{n}=\sum_{t=1}^{n} X_{t} . \\
Z_{n}(t)=\left\{\begin{array}{l}
T_{k} / \sqrt{n}, \text { if } t=k / n(k=0,1, \ldots, n) ; \\
\text { extended by linear interpolation in each interval } \\
{[[k-1] / n, k / n] .}
\end{array}\right.
\end{gathered}
$$

Put

$$
\begin{gathered}
\widetilde{X}_{t}=A \varepsilon_{t}=\left(\sum_{j=-\infty}^{\infty} \alpha_{j}\right) \varepsilon_{t}, \quad \widetilde{T}_{n}=\sum_{t=1}^{n} \widetilde{X}_{t} . \\
\widetilde{Z}_{n}(t)=\left\{\begin{array}{l}
\widetilde{T}_{k} / \sqrt{n}, \text { if } t=k / n(k=0,1, \ldots, n) ; \\
\text { extended by linear interpolation in each interval } \\
{[[k-1] / n, k / n] .}
\end{array}\right.
\end{gathered}
$$


Now we give the main results:

Theorem 3.1. Suppose $\widehat{\mathbb{E}}\left[\left(\varepsilon_{1}^{2}-b\right)^{+}\right]=0$ as $b \rightarrow \infty$. Then for all bounded continuous Lipschitz function $\varphi: \mathbb{R} \rightarrow \mathbb{R}$

$$
\lim _{n \rightarrow \infty} \widehat{\mathbb{E}}\left[\varphi\left(\frac{1}{A \sqrt{n}} T_{n}\right)\right]=\widetilde{\mathbb{E}}[\varphi(\xi)],
$$

where $\xi \sim \mathcal{N}\left(0 ;\left[\underline{\sigma}^{2}, \bar{\sigma}^{2}\right]\right)$ under $\widetilde{\mathbb{E}}$.

Theorem 3.2. Suppose $\widehat{\mathbb{E}}\left[\left(\varepsilon_{1}^{2}-b\right)^{+}\right]=0$ as $b \rightarrow \infty$. Then for all bounded continuous Lipschitz function $\varphi: C[0,1] \rightarrow \mathbb{R}$

$$
\widehat{\mathbb{E}}\left[\varphi\left(\frac{Z_{n}}{A}\right)\right] \rightarrow \widetilde{\mathbb{E}}[\varphi(W)],
$$

where $W(t)$ is a G-Brownian motion under $\widetilde{\mathbb{E}}$ with $W(1) \sim \mathcal{N}\left(0 ;\left[\underline{\sigma}^{2}, \bar{\sigma}^{2}\right]\right)$.

For proving Theorem 3.1 and Theorem 3.2, we need the following lemma.

Lemma 3.1. Let $\left\{\varepsilon_{j}, j \in \mathbb{Z}\right\}$ be a sequence of IID random variables on the sub-linear expectation space $(\Omega, \mathcal{H}, \widehat{\mathbb{E}})$. We further assume that $\widehat{\mathbb{E}}\left[\varepsilon_{1}\right]=\widehat{\mathbb{E}}\left[-\varepsilon_{1}\right]=0$, and $0<\bar{\sigma}^{2}=\widehat{\mathbb{E}}\left[\varepsilon_{1}^{2}\right], \underline{\sigma}^{2}=$ $\widehat{\mathcal{E}}\left[\varepsilon_{1}^{2}\right]$. If $\sum_{j=-\infty}^{\infty}\left|\alpha_{j}\right|<\infty$, then

$$
\frac{1}{\sqrt{n}} \widehat{\mathbb{E}}\left[\max _{1 \leq k \leq n}\left|\widetilde{T}_{k}-T_{k}\right|\right] \rightarrow 0,
$$

where $T_{k}, \widetilde{T}_{k}$ are defined as (5) and (7).

Proof $\quad$ Set $X_{t}=X_{t, 1}+X_{t, 2}, \quad \widetilde{X}_{t}=\widetilde{X}_{t, 1}+\widetilde{X}_{t, 2}, T_{k}=T_{k, 1}+T_{k, 2}, \widetilde{T}_{k}=\widetilde{T}_{k, 1}+\widetilde{T}_{k, 2}$, where $X_{t, 1}=\sum_{j=0}^{\infty} \alpha_{j} \varepsilon_{t-j} ; X_{t, 2}=\sum_{j=1}^{\infty} \alpha_{-j} \varepsilon_{t+j} ; \widetilde{X}_{t, 1}=\sum_{j=0}^{\infty} \alpha_{j} \varepsilon_{t} ; \widetilde{X}_{t, 2}=\sum_{j=1}^{\infty} \alpha_{-j} \varepsilon_{t} ; T_{k, 1}=$ $\sum_{t=1}^{k} X_{t, 1} ; T_{k, 2}=\sum_{t=1}^{k} X_{t, 2} ; \widetilde{T}_{k, 1}=\sum_{t=1}^{k} \widetilde{X}_{t, 1} ; \widetilde{T}_{k, 2}=\sum_{t=1}^{k} \widetilde{X}_{t, 2}$.

Note that (11) is equivalent to the following

$$
\begin{aligned}
& \frac{1}{\sqrt{n}} \widehat{\mathbb{E}}\left[\max _{1 \leq k \leq n}\left|\widetilde{T}_{k, 1}-T_{k, 1}\right|\right] \rightarrow 0, \\
& \frac{1}{\sqrt{n}} \widehat{\mathbb{E}}\left[\max _{1 \leq k \leq n}\left|\widetilde{T}_{k, 2}-T_{k, 2}\right|\right] \rightarrow 0 .
\end{aligned}
$$

We first want to prove (12), based on the above assumptions, we have

$$
\begin{aligned}
\widetilde{T}_{k, 1} & =\sum_{t=1}^{k}\left(\sum_{j=0}^{\infty} \alpha_{j}\right) \varepsilon_{t} \\
& =\sum_{t=1}^{k}\left(\sum_{j=0}^{k-t} \alpha_{j}\right) \varepsilon_{t}+\sum_{t=1}^{k}\left(\sum_{j=k-t+1}^{\infty} \alpha_{j}\right) \varepsilon_{t} \\
& =\sum_{t=1}^{k}\left(\sum_{j=0}^{t-1} \alpha_{j} \varepsilon_{t-j}\right)+\sum_{t=1}^{k}\left(\sum_{j=k-t+1}^{\infty} \alpha_{j}\right) \varepsilon_{t},
\end{aligned}
$$

thus

$$
\widetilde{T}_{k, 1}-T_{k, 1}=-\sum_{t=1}^{k}\left(\sum_{j=t}^{\infty} \alpha_{j} \varepsilon_{t-j}\right)+\sum_{t=1}^{k}\left(\sum_{j=k-t+1}^{\infty} \alpha_{j}\right) \varepsilon_{t}
$$




$$
\triangleq I_{k}+I I_{k}
$$

So

$$
\begin{aligned}
\frac{1}{\sqrt{n}} \widehat{\mathbb{E}}\left[\max _{1 \leq k \leq n}\left|\widetilde{T}_{k, 1}-T_{k, 1}\right|\right] & =\frac{1}{\sqrt{n}} \widehat{\mathbb{E}}\left[\max _{1 \leq k \leq n}\left|I_{k}+I I_{k}\right|\right] \\
& \leq \frac{1}{\sqrt{n}} \widehat{\mathbb{E}}\left[\max _{1 \leq k \leq n}\left|I_{k}\right|\right]+\frac{1}{\sqrt{n}} \widehat{\mathbb{E}}\left[\max _{1 \leq k \leq n}\left|I I_{k}\right|\right] .
\end{aligned}
$$

First to estimate $\frac{1}{\sqrt{n}} \widehat{\mathbb{E}}\left[\max _{1 \leq k \leq n}\left|I_{k}\right|\right]$, by(14), Lemma 2.1 and Lemma 2.2, we get

$$
\begin{aligned}
\frac{1}{\sqrt{n}} \widehat{\mathbb{E}}\left[\max _{1 \leq k \leq n}\left|I_{k}\right|\right] & =\frac{1}{\sqrt{n}} \widehat{\mathbb{E}}\left[\max _{1 \leq k \leq n} \mid \sum_{t=1}^{k}\left(\sum_{j=t}^{\infty} \alpha_{j} \varepsilon_{t-j}\right)\right] \\
& \leq \frac{1}{\sqrt{n}} \sum_{j=1}^{\infty}\left|\alpha_{j}\right|\left(\widehat{\mathbb{E}}\left[\max _{1 \leq k \leq n}\left|\sum_{t=1}^{j \wedge k} \varepsilon_{t-j}\right|^{2}\right]\right)^{\frac{1}{2}} \\
& \leq \sum_{j=1}^{\infty}\left|\alpha_{j}\right|\left(\widehat{\mathbb{E}}\left[\varepsilon_{1}^{2}\right]\left(\frac{j \wedge n}{n}\right)\right)^{\frac{1}{2}} \\
& \leq \widehat{\mathbb{E}}\left[\varepsilon_{1}^{2}\right]\left(\sum_{j=1}^{n}\left|\alpha_{j}\right|\left(\frac{j}{n}\right)^{\frac{1}{2}}+\sum_{j=n+1}^{\infty}\left|\alpha_{j}\right|\right) \\
& =o(1) .
\end{aligned}
$$

Next to estimate $\frac{1}{\sqrt{n}} \widehat{\mathbb{E}}\left[\max _{1 \leq k \leq n}\left|I I_{K}\right|\right]$, we write

$$
I I_{k}=I I_{k 1}+I I_{k 2}
$$

so we have

where

$$
\begin{aligned}
\frac{1}{\sqrt{n}} \widehat{\mathbb{E}}\left[\max _{1 \leq k \leq n}\left|I I_{k}\right|\right] & =\frac{1}{\sqrt{n}} \widehat{\mathbb{E}}\left[\max _{1 \leq k \leq n}\left|I I_{k 1}+I I_{k 2}\right|\right] \\
& \leq \frac{1}{\sqrt{n}} \widehat{\mathbb{E}}\left[\max _{1 \leq k \leq n}\left|I I_{k 1}\right|\right]+\frac{1}{\sqrt{n}} \widehat{\mathbb{E}}\left[\max _{1 \leq k \leq n}\left|I I_{k 2}\right|\right]
\end{aligned}
$$

and

$$
I I_{k 1}=\alpha_{1} \varepsilon_{k}+\alpha_{2}\left(\varepsilon_{k}+\varepsilon_{k-1}\right)+\cdots+\alpha_{k}\left(\varepsilon_{k}+\cdots+\varepsilon_{1}\right),
$$

and

$$
I I_{k 2}=\left(\alpha_{k+1}+\alpha_{k+2}+\cdots\right)\left(\varepsilon_{k}+\cdots+\varepsilon_{1}\right),
$$

and let $p_{n}$ be a sequence of positive integers such that

$$
p_{n} \rightarrow \infty \text { and } p_{n} / n \rightarrow 0 \text { as } n \rightarrow \infty,
$$

then

$$
\begin{aligned}
\frac{1}{\sqrt{n}} \widehat{\mathbb{E}}\left[\max _{1 \leq k \leq n}\left|I I_{k 2}\right|\right] \leq & \left(\sum_{j=0}^{\infty}\left|\alpha_{j}\right|\right) \frac{1}{\sqrt{n}} \widehat{\mathbb{E}}\left[\max _{1 \leq k \leq p_{n}}\left|\varepsilon_{1}+\cdots+\varepsilon_{k}\right|\right] \\
& +\left(\sum_{j>p_{n}}\left|\alpha_{j}\right|\right) \frac{1}{\sqrt{n}} \widehat{\mathbb{E}}\left[\max _{1 \leq k \leq n}\left|\varepsilon_{1}+\cdots+\varepsilon_{k}\right|\right] \\
\leq & \left(\sum_{j=0}^{\infty}\left|\alpha_{j}\right|\right) \frac{1}{\sqrt{n}}\left(\widehat{\mathbb{E}}\left[\max _{1 \leq k \leq p_{n}}\left|\varepsilon_{1}+\cdots+\varepsilon_{k}\right|^{2}\right]\right)^{\frac{1}{2}}
\end{aligned}
$$




$$
\begin{aligned}
& +\left(\sum_{j>p_{n}}\left|\alpha_{j}\right|\right) \frac{1}{\sqrt{n}}\left(\widehat{\mathbb{E}}\left[\max _{1 \leq k \leq n}\left|\varepsilon_{1}+\cdots+\varepsilon_{k}\right|^{2}\right]\right)^{\frac{1}{2}} \\
\leq & \left(\sum_{j=0}^{\infty}\left|\alpha_{j}\right|\right)\left(\widehat{\mathbb{E}}\left[\varepsilon_{1}^{2}\right]\left(p_{n} / n\right)\right)^{\frac{1}{2}}+\left(\sum_{j>p_{n}}\left|\alpha_{j}\right|\right) \frac{1}{\sqrt{n}}\left(n \widehat{\mathbb{E}}\left[\varepsilon_{1}^{2}\right]\right)^{\frac{1}{2}} \\
= & o(1) .
\end{aligned}
$$

It remains to show that

$$
\frac{1}{\sqrt{n}} \widehat{\mathbb{E}}\left[\max _{1 \leq k \leq n}\left|I I_{k 1}\right|\right]=o(1)
$$

For each $m \geq 1$, define

$$
I I_{k 1, m}=b_{1} \varepsilon_{k}+b_{2}\left(\varepsilon_{k}+\varepsilon_{k-1}\right)+\cdots+b_{k}\left(\varepsilon_{k}+\cdots+\varepsilon_{1}\right),
$$

where $b_{k}=\alpha_{k}$ for $k \leq m$ and $b_{k}=0$ otherwise.

First note that, for each $m$,

$$
\frac{1}{\sqrt{n}} \max _{1 \leq k \leq n}\left|I I_{k 1, m}\right| \leq \frac{1}{\sqrt{n}}\left(\left|\alpha_{1}\right|+\left|\alpha_{2}\right|+\cdots+\left|\alpha_{m}\right|\right)\left(\left|\varepsilon_{1}\right|+\left|\varepsilon_{2}\right|+\cdots+\left|\varepsilon_{m}\right|\right),
$$

then by Lemma 2.2 we have

Note

$$
\begin{aligned}
\frac{1}{\sqrt{n}} \widehat{\mathbb{E}}\left[\max _{1 \leq k \leq n}\left|I I_{k 1, m}\right|\right] & \leq \frac{2}{\sqrt{n}} \sum_{j=1}^{m}\left|\alpha_{j}\right| \widehat{\mathbb{E}}\left[\left|\varepsilon_{1}\right|+\left|\varepsilon_{2}\right|+\cdots+\left|\varepsilon_{m}\right|\right] \\
& \leq \frac{2}{\sqrt{n}} \sum_{j=1}^{m}\left|\alpha_{j}\right| m\left(\widehat{\mathbb{E}}\left[\varepsilon_{1}^{2}\right]\right)^{\frac{1}{2}}
\end{aligned}
$$

Since

$$
\begin{aligned}
& \frac{1}{\sqrt{n}} \max _{1 \leq k \leq n}\left|I I_{k 1, m}-I I_{k 1}\right| \\
= & \frac{1}{\sqrt{n}} \max _{1 \leq k \leq n}\left|\sum_{i=1}^{k}\left(\alpha_{i}-b_{i}\right)\left(\varepsilon_{k}+\cdots+\varepsilon_{k-i+1}\right)\right| .
\end{aligned}
$$

$$
\left|\sum_{i=1}^{k}\left(\alpha_{i}-b_{i}\right)\left(\varepsilon_{k}+\cdots+\varepsilon_{k-i+1}\right)\right|= \begin{cases}0, & k \leq m \\ \left|\sum_{i=m+1}^{k} \alpha_{i}\left(\varepsilon_{k}+\cdots+\varepsilon_{k-i+1}\right)\right|, & \text { otherwise }\end{cases}
$$

then

$$
\begin{aligned}
& \frac{1}{\sqrt{n}} \max _{1 \leq k \leq n}\left|I I_{k 1, m}-I I_{k 1}\right| \\
= & \frac{1}{\sqrt{n}} \max _{1 \leq k \leq n}\left|\sum_{i=1}^{k}\left(\alpha_{i}-b_{i}\right)\left(\varepsilon_{k}+\cdots+\varepsilon_{k-i+1}\right)\right| \\
\leq & \frac{1}{\sqrt{n}} \max _{m<k \leq n}\left(\sum_{i=m+1}^{k}\left|\alpha_{i}\right|\left|\varepsilon_{k}+\cdots+\varepsilon_{k-i+1}\right|\right) \\
\leq & \frac{1}{\sqrt{n}} \max _{m<k \leq n} \sum_{i=m+1}^{k}\left|\alpha_{i}\right| \max _{m<i \leq k}\left|\varepsilon_{k}+\cdots+\varepsilon_{k-i+1}\right|
\end{aligned}
$$




$$
\begin{aligned}
& \leq \frac{1}{\sqrt{n}} \sum_{i>m}\left|\alpha_{i}\right| \max _{m<k \leq n} \max _{m<i \leq k}\left(\left|\varepsilon_{1}+\cdots+\varepsilon_{k}\right|+\left|\varepsilon_{1}+\cdots+\varepsilon_{k-i}\right|\right) \\
& \leq \frac{1}{\sqrt{n}} \sum_{i>m}\left|\alpha_{i}\right|\left(\max _{m<k \leq n}\left|\varepsilon_{k}+\cdots+\varepsilon_{1}\right|+\max _{m<k \leq n} \max _{m<i \leq k}\left|\varepsilon_{1}+\cdots+\varepsilon_{k-i}\right|\right) \\
& \leq \frac{1}{\sqrt{n}} \sum_{i>m}\left|\alpha_{i}\right|\left(\max _{1<j \leq n}\left|\varepsilon_{1}+\cdots+\varepsilon_{j}\right|+\max _{1 \leq j \leq n}\left|\varepsilon_{1}+\cdots+\varepsilon_{j}\right|\right) \\
& \leq 2 \frac{1}{\sqrt{n}} \sum_{i>m}\left|\alpha_{i}\right| \max _{1 \leq j \leq n}\left|\varepsilon_{1}+\cdots+\varepsilon_{j}\right| .
\end{aligned}
$$

Therefore

$$
\begin{aligned}
\frac{1}{\sqrt{n}} \widehat{\mathbb{E}}\left[\max _{1 \leq k \leq n}\left|I I_{k 1, m}-I I_{k 1}\right|\right] & \leq C \frac{1}{\sqrt{n}} \sum_{i>m}\left|\alpha_{i}\right| \widehat{\mathbb{E}}\left[\max _{1 \leq j \leq n}\left|\varepsilon_{1}+\cdots+\varepsilon_{j}\right|\right] \\
& \leq C \frac{1}{\sqrt{n}}\left(\sum_{i>m}\left|\alpha_{i}\right|\right)\left(\widehat{\mathbb{E}}\left[\max _{1 \leq j \leq n}\left|\varepsilon_{1}+\cdots+\varepsilon_{j}\right|^{2}\right]\right)^{\frac{1}{2}} \\
& \leq C \frac{1}{\sqrt{n}}\left(\sum_{i>m}\left|\alpha_{i}\right|\right)\left(n \widehat{\mathbb{E}}\left[\varepsilon_{1}^{2}\right]\right)^{\frac{1}{2}} \\
& =C\left(\sum_{i>m}\left|\alpha_{i}\right|\right) .
\end{aligned}
$$

Let $m=\left[n^{\frac{1}{3}}\right]$, from (18) and (19), we obtain (17). Combining with (16) and (17), we have

$$
\frac{1}{\sqrt{n}} \widehat{\mathbb{E}}\left[\max _{1 \leq k \leq n}\left|I I_{k}\right|\right] \leq \frac{1}{\sqrt{n}} \widehat{\mathbb{E}}\left[\max _{1 \leq k \leq n}\left|I I_{k 1}\right|\right]+\frac{1}{\sqrt{n}} \widehat{\mathbb{E}}\left[\max _{1 \leq k \leq n}\left|I I_{k 2}\right|\right]=o(1),
$$

and together with (15), then follows (12), the proof method of (13) is similar to that of (12). Therefore Lemma 3.1 is proved.

Proof of Theorem 3.1. In order to prove (9), it suffices to prove

$$
\left|\widehat{\mathbb{E}}\left[\varphi\left(\frac{T_{n}}{A \sqrt{n}}\right)\right]-\widetilde{\mathbb{E}}[\varphi(\xi)]\right| \rightarrow 0, \quad n \rightarrow \infty,
$$

by the Remark 2.1(ii), for $\forall \varphi \in C_{b, L i p}\left(\mathbb{R}^{n}\right)$ we have

$$
\begin{aligned}
\left|\widehat{\mathbb{E}}\left[\varphi\left(\frac{T_{n}}{A \sqrt{n}}\right)\right]-\widetilde{\mathbb{E}}[\varphi(\xi)]\right| & =\left|\widehat{\mathbb{E}}\left[\varphi\left(\frac{T_{n}}{A \sqrt{n}}\right)\right]-\widehat{\mathbb{E}}\left[\varphi\left(\frac{\widetilde{T}_{n}}{A \sqrt{n}}\right)\right]+\widehat{\mathbb{E}}\left[\varphi\left(\frac{\widetilde{T}_{n}}{A \sqrt{n}}\right)\right]-\widetilde{\mathbb{E}}[\varphi(\xi)]\right| \\
& \leq\left|\widehat{\mathbb{E}}\left[\varphi\left(\frac{T_{n}}{A \sqrt{n}}\right)\right]-\widehat{\mathbb{E}}\left[\varphi\left(\frac{\widetilde{T}_{n}}{A \sqrt{n}}\right)\right]\right|+\left|\widehat{\mathbb{E}}\left[\varphi\left(\frac{\widetilde{T}_{n}}{A \sqrt{n}}\right)\right]-\widetilde{\mathbb{E}}[\varphi(\xi)]\right| \\
& \leq\left|\widehat{\mathbb{E}}\left[\varphi\left(\frac{T_{n}}{A \sqrt{n}}\right)-\varphi\left(\frac{\widetilde{T}_{n}}{A \sqrt{n}}\right)\right]\right|+\left|\widehat{\mathbb{E}}\left[\varphi\left(\frac{\widetilde{T}_{n}}{A \sqrt{n}}\right)\right]-\widetilde{\mathbb{E}}[\varphi(\xi)]\right| \\
& \left.\leq C \frac{1}{\sqrt{n}} \widehat{\mathbb{E}}\left[\left|T_{n}-\widetilde{T}_{n}\right|\right]+\mid \widehat{\mathbb{E}}\left[\varphi\left(\frac{\widetilde{T}_{n}}{A \sqrt{n}}\right)\right]-\widetilde{\mathbb{E}}[\varphi(\xi)]\right] .
\end{aligned}
$$

Since $\widetilde{X}_{t}=A \varepsilon_{t}=\left(\sum_{j=-\infty}^{\infty} \alpha_{j}\right) \varepsilon_{t}, \widetilde{T}_{n}=\sum_{t=1}^{n} \widetilde{X}_{t}$, by Theorem 2.1, we have $\mid \widehat{\mathbb{E}}\left[\varphi\left(\frac{\widetilde{T}_{n}}{A \sqrt{n}}\right)\right]-$ $\widetilde{\mathbb{E}}[\varphi(\xi)] \mid \rightarrow 0$. We can just prove $\frac{1}{\sqrt{n}} \widehat{\mathbb{E}}\left|\widetilde{T}_{n}-T_{n}\right| \rightarrow 0$ to get $(21)$, and the proof of Theorem 3.1 is completed through Lemma 3.1 . 
Proof of Theorem 3.2. By the Remark 2.1(ii), for $\forall \varphi \in C_{b, L i p}\left(\mathbb{R}^{n}\right)$ we have

$$
\begin{aligned}
\left|\widehat{\mathbb{E}}\left[\varphi\left(\frac{Z_{n}}{A}\right)\right]-\widetilde{\mathbb{E}}[\varphi(W)]\right| & =\left|\widehat{\mathbb{E}}\left[\varphi\left(\frac{Z_{n}}{A}\right)\right]-\widehat{\mathbb{E}}\left[\varphi\left(\frac{\widetilde{Z}_{n}}{A}\right)\right]+\widehat{\mathbb{E}}\left[\varphi\left(\frac{\widetilde{Z}_{n}}{A}\right)\right]-\widetilde{\mathbb{E}}[\varphi(W)]\right| \\
& \leq C \widehat{\mathbb{E}}\left[\sup _{0 \leq t \leq 1}\left|Z_{n}(t)-\widetilde{Z}_{n}(t)\right|\right]+\left|\widehat{\mathbb{E}}\left[\varphi\left(\frac{\widetilde{Z}_{n}}{A}\right)\right]-\widetilde{\mathbb{E}}[\varphi(W)]\right| \\
& \leq C \frac{1}{\sqrt{n}} \widehat{\mathbb{E}}\left[\max _{1 \leq k \leq n}\left|\widetilde{T}_{k}-T_{k}\right|\right]+\left|\widehat{\mathbb{E}}\left[\varphi\left(\frac{\widetilde{Z}_{n}}{A}\right)\right]-\widetilde{\mathbb{E}}[\varphi(W)]\right| .
\end{aligned}
$$

Since $\widetilde{X}_{t}=A \varepsilon_{t}$, note that $\left\{\widetilde{X_{t}}\right\}$ satisfies conditions of Theorem 2.1. From (6) and (8), it implies that Theorem 2.2 holds for the sequence $\left\{\widetilde{Z}_{n}\right\}$. Then by Theorem 2.2 we obtain

By Lemma 3.1 we have

$$
\left|\widehat{\mathbb{E}}\left[\varphi\left(\frac{\widetilde{Z}_{n}}{A}\right)\right]-\widetilde{\mathbb{E}}[\varphi(W)]\right| \rightarrow 0, \quad n \rightarrow \infty .
$$

$$
\frac{1}{\sqrt{n}} \widehat{\mathbb{E}}\left[\max _{1 \leq k \leq n}\left|\widetilde{T}_{k}-T_{k}\right|\right] \rightarrow 0, \quad n \rightarrow \infty .
$$

Combining (22), (23) and (24), it follows that

The proof of Theorem 3.2 now completes.

$$
\left|\widehat{\mathbb{E}}\left[\varphi\left(\frac{Z_{n}}{A}\right)\right]-\widetilde{\mathbb{E}}[\varphi(W)]\right| \rightarrow 0, \quad n \rightarrow \infty .
$$

Remark 3.1. Obviously, if in Lemma3.1, Theorem 3.1 and Theorem 3.2 the sub-linear expectations were substituted by usual expectations, our results still holds. Remark 3.4 of the Peng [22] show that if $\bar{\sigma}^{2}=\underline{\sigma}^{2}$ then the G-normal distribution becomes the classical normal distribution, and the CLT (see Theorem 3.1 in Chapter II of Peng [22]) in the form of " $\lim _{n \rightarrow \infty} \widehat{\mathbb{E}}\left[\varphi\left(\frac{1}{\sqrt{n}} \sum_{i=1}^{n} \varepsilon_{i}\right)\right]=\widetilde{\mathbb{E}}[\varphi(\xi)] "$ becomes a classical central limit theorem. This implies that our results can also be regarded as an extension of CLT of linear process generated by IID random variable sequence in classical framework.

Remark 3.2. Note that if $\alpha_{0}=1, \alpha_{j}=0, j \neq 0$, Theorem 3.1 can be regarded as Theorem 2.1, Theorem 3.2 can be regarded as Theorem 2.2.

Open Access This article is licensed under a Creative Commons Attribution 4.0 International License, which permits use, sharing, adaptation, distribution and reproduction in any medium or format, as long as you give appropriate credit to the original author(s) and the source, provide a link to the Creative Commons licence, and indicate if changes were made. The images or other third party material in this article are included in the articles Creative Commons licence, unless indicated otherwise in a credit line to the material. If material is not included in the articles Creative Commons licence and your intended use is not permitted by statutory regulation or exceeds the permitted use, you will need to obtain permission directly from the copyright holder. To view a copy of this licence, visit http://creativecomm ons.org/licenses/by/4.0/. 


\section{References}

[1] A Linero, A Rosalsky. On the Toeplitz lemma, convergence in probability, and mean convergence, Stochastic Analysis and Applications, 2013, 31(4): 684-694.

[2] L Denis, M Hu, S Peng. Function spaces and capacity related to a sublinear expectation: application to G-Brownian Motion Pathes, Potential Analysis, 2011, 34(2); 139-161

[3] D Rokhlin. Central limit theorem under variance uncertainty, Electron Commun Probab, 2015, 20(66): 1-10.

[4] E Hannan. Multivariate time series, Wiley, New York, 1970.

[5] F Hu, D Zhang. Central limit theorem for capacities, Comples Rendus Mathematique, 2010, 348(19-20): 1111-1114.

[6] F Hu. Moment bounds for IID sequences under sublinear expectations, Science China Mathematics, 2011, 54(10): 2155-2160.

[7] I Gilboa. Expected utility with purely subjective non-additive probabilities, Journal of Mathematical Economics, 1987, 16(1): 65-88.

[8] J Xu, L Zhang. Three series theorem for independent random variables under sub-linear expectations with applications, Acta Mathematica Sinica, 2019, 35(2): 172-184.

[9] L Denis, C Martini. A theoretical framework for the pricing of contingent claims in the presence of model uncertainty, Annals of Applied Probability, 2006, 16(2): 827-852.

[10] L Zhang. Exponential inequalities under the sub-linear expectations with applications to laws of the iterated logarithm, Science China Mathematics, 2016, 59(12): 2503-2526.

[11] L Zhang. Lindeberg's central limit theorems for martingale like sequences under sublinear expectations, Science China Mathematics, 2020, DOI: 10.1007/s11425-018-9556-7, (in press).

[12] L Zhang. Donsker's invariance principle under the sub-linear expectation with an application to chung's law of the iterated logarithm, Communications in Mathematics and Statistics, 2015, 3(2): 187-214.

[13] L Zhang. Rosenthal's inequalities for independent and negatively dependent random variables under sub-linear expectations with applications, Science China Mathematics, 2016, 59(4): 751-768.

[14] M Marinacci. Limit laws for non-additive probabilities and their frequentist interpretation, Journal of Economic Theory, 2004, 84(2): 145-195.

[15] $\mathrm{P} \mathrm{Wu}, \mathrm{Z}$ Chen. Invariance principles for the law of the iterated logarithm under Gframework, Science China Mathematics, 2015, 58(6): 1251-1264. 
[16] S Peng. Backward SDE and related G-Expectation, Backward Stochastic Differential Equations, 1997: 141-159.

[17] S Peng. Monotonic limit theorem of BSDE and nonlinear decomposition theorem of DoobMeyers type, Probability Theory and Related Fields, 1999, 113(4): 473-499.

[18] S Peng. G-Expectation, G-Brownian motion and related stochastic calculus of Ito's type, Stochastic Analysis and Applications, 2006, 2(4): 541-567.

[19] S Peng. Law of large numbers and central limit theorem under nonlinear expectations, Probability, Uncertainty and Quantitative Risk, 2019, DOI: 10.1186/s41546-019-0038-2.

[20] S Peng. Multi-dimensional G-Brownian motion and related stochastic calculus under GExpectation, Stochastic Processes and their Applications, 2008, 118(12): 2223-2253.

[21] S Peng. Survey on normal distributions, central limit theorem, Brownian motion and the related stochastic calculus under sublinear expectations, Science China Mathematics, 2009, 52(7): 1391-1411.

[22] S Peng. Nonlinear Expectations and Stochastic Calculus under Uncertainty, First Edition, 2010.

[23] X Li. A central limit theorem for m-dependent random variables under sublinear expectations, Acta Mathematicae Applicatae Sinica, English Series, 2015, 31(2): 435-444.

[24] Z Hu, L Zhou. Multi-dimensional central limit theorems and laws of large numbers under sublinear expectations, Acta Mathematica Sinica, English Series, 2015, 31(2): 305-318.

School of Mathematics, Jilin University, Changchun 130012, China.

Email: zyong2661@jlu.edu.cn 